\title{
Is Early Enteral Nutrition Initiated Within 24 Hours Better for the Postoperative Course in Esophageal Cancer Surgery?
}

\author{
Naoko Manba ${ }^{a}$, Yu Koyamaa, b, Shin-ichi Kosugi ${ }^{a}$, Takashi Ishikawa ${ }^{a}$, Hiroshi Ichikawa ${ }^{a}$, \\ Masahiro Minagawa ${ }^{a}$, Takashi Kobayashi ${ }^{a}$, Toshifumi Wakai ${ }^{a}$
}

\begin{abstract}
Background: Early enteral nutrition within $24 \mathrm{~h}$ after surgery has become a recommended procedure. In the present study, we retrospectively examined whether initiating EN within $24 \mathrm{~h}$ after esophagectomy improves the postoperative course.
\end{abstract}

Methods: Among 103 patients who underwent thoracic esophagectomy for esophageal cancer, we enrolled the cases in which EN was initiated within $72 \mathrm{~h}$ after surgery. The patients were divided into two groups: EN started within $24 \mathrm{~h}$ (Group D1) and EN started at 24 - 72 h (Group D2-3). Clinical factors including days for first fecal passage, dose of postoperative albumin infusion, difference in serum albumin between pre- and postoperation, incidence of postoperative infection, and use of total parenteral nutritionwere compared. Statistical analyses were performed by the Mann-Whitney $U$ test and Chi square test, with significance defined as $\mathrm{P}<0.05$.

Results: There was no significant difference between the groups in clinical factors. While pneumonia was significantly more frequent in Group D1 than in Group D2-3 $(\mathrm{P}=0.0308)$, the frequency of infectious complications was comparable between the groups.

Conclusion: Initiating EN within $24 \mathrm{~h}$ showed no advantage for the postoperative course in esophageal cancer, and thus EN should be scheduled within $24-72 \mathrm{~h}$, based on the patient condition.

Keywords: Early enteral nutrition; Esophageal cancer; Infectious complication; Pneumonia

\footnotetext{
Manuscript accepted for publication November 19, 2013

${ }^{a}$ Division of Digestive and General Surgery, Niigata University Graduate School of Medical and Dental Sciences, Niigata, Japan

${ }^{\mathrm{b}}$ Corresponding author: Yu Koyama, Division of Digestive and General Surgery, Niigata University Graduate School of Medical and Dental Sciences, 1-757 Asahimachi, Niigata 951-8510, Japan.

Email: yukmy@med.niigata-u.ac.jp
}

doi: http://dx.doi.org/10.4021/jocmr1665w

\section{Introduction}

Several studies have advocated early enteral nutrition (EN) in various patient groups including critically ill patients [1-3], acutely hospitalized medical patients [4, 5], burns patients [6-9], trauma patients $[10,11]$, and septic patients $[12,13]$. Moreover, patients undergoing major surgery have gained proven advantage from EN started at an earlier stage after surgery [14-20]. These studies have demonstrated several advantages of early EN initiated postoperatively as follows: lower incidence of septic complications [14], shorter hospital stay, and decreased weight loss [18]. Classically, the term "early" was defined as EN started within 3 days after admission or surgery [9]; however, "early" is more recently considered as EN started within 24 - $48 \mathrm{~h}$ after admission or surgery [1]. Further, EN started within $24 \mathrm{~h}$ after surgery or trauma has been shown some benefits such as reduction in mortality, septic complication, and life-threatening complications [11, 21-23]. Transthoracic esophagectomy with 3-field lymphadenectomy (TTE-3FL) for esophageal cancer is one of the most invasive procedures among gastrointestinal surgeries, and patients undergoing TTE-3FL are unable to gain nutrition by mouth within the first few days after surgery. Thus, postoperative EN and/or parenteral nutrition have become routine management in such cases, and recent studies demonstrated that EN initiated within 24 - $48 \mathrm{~h}$ after esophagectomy reduced the length of hospital stay [24, 25], postoperative morbidity [20], and the rate of life-threatening complications [22]. Moreover, some clinical guidelines on EN for surgical patient have also recommended early EN initiated within $24 \mathrm{~h}$ after gastrointestinal surgery, including esophagectomy [26]. However, almost all of these studies described the superiority of EN initiated within $24 \mathrm{~h}$ in comparison with total parenteral nutrition (TPN), thus it remains unclear whether EN initiated within $24 \mathrm{~h}$ is more beneficial in comparison with EN initiated $24-72 \mathrm{~h}$ after trauma or surgery, including esophagectomy. Moreover, because some studies could not show any clinical benefits with routine postoperative EN after esophagectomy [27-29], the validity of early EN after esophagectomy, especially within $24 \mathrm{~h}$, is still controversial [30]. 
Table 1. Pre- and Peri-Operative Clinicopathological Feature

\begin{tabular}{|c|c|c|c|c|}
\hline & Total & D1 $(n=15)$ & D2-3 $(n=27)$ & P value (Mann-Whitney test) \\
\hline Age (years) & $61.5 \pm 6.6$ & $60.5 \pm 6.3$ & $62.1 \pm 6.8$ & 0.5373 \\
\hline BMI $\left(\mathrm{kg} / \mathrm{m}^{2}\right)$ & $21.8 \pm 3.1$ & $20.9 \pm 2.4$ & $22.4 \pm 3.4$ & 0.1246 \\
\hline Body weight (kg) & $57.7 \pm 9.8$ & $55.1 \pm 9.5$ & $59.1 \pm 9.9$ & 0.1488 \\
\hline Albumin (mg/dL) & $4.0 \pm 0.4$ & $3.9 \pm 0.5$ & $4.0 \pm 0.3$ & 0.6600 \\
\hline Operative time (min) & $470 \pm 83$ & $475 \pm 83$ & $467 \pm 85$ & 0.9060 \\
\hline Blood loss (mL) & $676 \pm 280$ & $679 \pm 273$ & $673 \pm 290$ & 0.7528 \\
\hline & & & & $P$ value (Chi square test) \\
\hline Sex & & & & 0.8950 \\
\hline Male & 36 & 13 & 23 & \\
\hline Female & 6 & 2 & 4 & \\
\hline Stage & & & & 0.1536 \\
\hline 0 & 2 & 1 & 1 & \\
\hline I & 15 & 4 & 11 & \\
\hline IIA & 7 & 1 & 6 & \\
\hline IIB & 2 & 1 & 1 & \\
\hline III & 8 & 6 & 2 & \\
\hline IVA & 3 & 0 & 3 & \\
\hline IVB & 5 & 2 & 3 & \\
\hline Preop. chemotherapy & & & & 0.0129 \\
\hline No & 22 & 4 & 18 & \\
\hline Yes & 20 & 11 & 9 & \\
\hline Postop. TPN use & & & & 0.2348 \\
\hline No & 32 & 13 & 19 & \\
\hline Yes & 10 & 2 & 8 & \\
\hline
\end{tabular}

TPN: total parenteral nutrition.

The aim of the present retrospective study was to examine whether early EN initiated within $24 \mathrm{~h}$ after esophagectomy is superior to EN initiated $24-72 \mathrm{~h}$ in terms of the postoperative course.

\section{Materials and Methods}

\section{Patient selection}

Patients who underwent TTE-3FL for esophageal cancer at
Niigata University Medical and Dental Hospital during 1996 - 2010 (103 patients in total) were entered into the present retrospective chart review. Among these cases, we analyzed the data from 42 patients for whom EN was initiated within 3 postoperative days. The study protocol was approval by the Institutional Review Board for Clinical Research. The patients were divided into two groups based on the start date of EN administration as follows: Group D1 comprised patients started on EN within $24 \mathrm{~h}$ after surgery, and Group D2-3 comprised patients started on EN within 24 - $72 \mathrm{~h}$ after TTE3FL. The EN was started with an initial dose of $200-250$ 
Table 2. Postoperative Complications and Mortality

\begin{tabular}{lllll}
\hline & Total & D1 (n= 15) & D2-3 (n= 27) & P value \\
& & & & \\
\hline Postop. complications & 36 & 13 & 23 & 0.8954 \\
Non-infectious & 21 & 8 & 13 & 0.7474 \\
$\quad$ Recurrent nerve palsy & 22 & 8 & 14 & 0.9266 \\
Anastomotic dehiscence & 14 & 5 & 9 & 0.9999 \\
Tracheal damage & 1 & 1 & 0 & 0.6657 \\
Aortic rupture & 1 & 1 & 0 & 0.6657 \\
Infectious & 17 & 6 & 11 & 0.9626 \\
Pneumonia & 7 & 5 & 2 & 0.0308 \\
Wound infection & 7 & 3 & 4 & 0.6657 \\
Sepsis & 3 & 0 & 3 & 0.4749 \\
Mortality & 1 & 1 & 0 & 0.6657 \\
\hline
\end{tabular}

$\mathrm{mL}$ of oligomeric or polymeric formula $(1 \mathrm{kcal} / \mathrm{mL})$ under $10-20 \mathrm{~mL} / \mathrm{h}$ from the jejunostomy. In both groups, the EN dose was gradually increased every $12-24 \mathrm{~h}$ if there were no problems related to the $\mathrm{EN}$, to reach a maximum dose at day 5 - 6 after starting EN. Peripheral intravenous infusions of $4.3 \%$ glucose with electrolyte solutions were also supplied in both groups. TPN was introduced if the EN could not be started by postoperative day 5 .

\section{Clinicopathological assessment}

Clinicopathological factors were compared between the groups as follows: age, sex, tumor stage according to the tumor-node metastasis classification of the International Union Against Cancer (6th edition), days for first fecal passage, the dose of postoperative albumin infusion used, difference in serum albumin values between day 7 and the presurgical assessment $(\Delta \mathrm{alb})$, duration of systematic inflammatory response syndrome (SIRS), incidence of postoperative complications, and use of TPN. SIRS was diagnosed according to the criteria of the ACCP-SCCM Consensus Conference Committee [28]. For the diagnosis, at least two of the following criteria had to be fulfilled: systolic blood pressure $<90$ $\mathrm{mmHg}$, tachycardia $>90 /$ minutes, respiratory rate $>20 / \mathrm{min}-$ utes or peripheral arterial $\mathrm{CO}_{2}$ tension $\left(\mathrm{PaCO}_{2}\right)<32 \mathrm{mmHg}$, temperature $>38.0^{\circ} \mathrm{C}$ or $<36.0^{\circ} \mathrm{C}$, leukocytosis $>12,000 /$ $\mu \mathrm{L}$ or leukopenia $<4,000 / \mu \mathrm{L}$ or $10 \%$ immature (band) forms.

Postoperative complications were also retrospectively reviewed from patient records, and complications were classified as either non-infectious or infectious. In this study, a mechanical complication was defined as that directly due to a failure in the surgical procedure, and an infectious com- plication was one accompanying infection. Recurrent nerve palsy was assessed clinically and endoscopically, and subclinical recurrent nerve palsy was also judged as positive.

\section{Statistical analysis}

The statistical analyses were performed using the MannWhitney $U$ test and Chi square test. Statistical significance was defined as $\mathrm{P}<0.05$.

\section{Results}

\section{Pre- and peri-operative clinicopathological features}

Among the 42 patients who received early EN, 15 patients were categorized into Group D1, and 27 patients were categorized into Group D2-3 (Table 1). There was no significant difference in mean age, sex, preoperative nutritional conditions expressed by body mass index (BMI), body weight, or serum albumin values between Group D1 and Group D2-3. Tumor stage was also comparable between the groups; however, preoperative chemotherapy was significantly more frequent in Group D1 patients than in Group D2-3 $(\mathrm{P}=0.0129)$. Postoperative TPN use was comparable between the groups.

\section{Postoperative complications and mortality}

Postoperative complications were observed in 36 patients $(85.7 \%)$, but there was no significant difference in morbidity between Group D1 and Group D2-3. Further analysis was performed by dividing the complications into non-in- 
Table 3. Postoperative Outcomes of Early EN Initiated Within $24 \mathrm{~h}$ and During 24 - $72 \mathrm{~h}$

\begin{tabular}{lllll}
\hline & Total & D1 (n= 15) & D2-3 (n=27) & P value \\
\hline & & & & \\
\hline EN calorie (kcal/kg) & $28.4 \pm 8.5$ & $26.5 \pm 8.1$ & $29.4 \pm 8.6$ & 0.3249 \\
First fecal passage (day) & $6.5 \pm 2.3$ & $4.8 \pm 1.7$ & $5.5 \pm 1.6$ & 0.2535 \\
Albumin infusion (mL) & $83.1 \pm 61.7$ & $79.3 \pm 66.9$ & $85.0 \pm 59.8$ & 0.8031 \\
$\Delta$ albumin (mg/dL) & $-1.18 \pm 0.5$ & $-1.3 \pm 0.6$ & $-1.1 \pm 0.4$ & 0.4833 \\
SIRS duration (day) & $4.0 \pm 5.1$ & $5.8 \pm 8.3$ & $3.0 \pm 2.0$ & 0.9525 \\
Respirator duration (day) & $3.7 \pm 7.3$ & $5.9 \pm 11.9$ & $2.6 \pm 1.6$ & 0.5636 \\
LOH (day) & $54.2 \pm 52.0$ & $68.9 \pm 74.0$ & $46.0 \pm 33.4$ & 0.6087 \\
\hline
\end{tabular}

$\Delta$ albumin: difference in serum albumin values between postoperative day 7 and pre-operation; SIRS: systematic inflammatory response syndrome; LOH: length of hospital stay.

fectious and infectious. The groups showed no difference in non-infectious complications. Frequency of postoperative infectious complications were also comparable between the groups; however, pneumonia was significantly more frequent in Group D1 compared with Group D2-3 ( $\mathrm{P}=0.0308)$. The mortality rate among all patients was comparable (Table 2).

\section{Postoperative outcomes between Group D1 and D2-3}

There was no significant difference in postoperative EN calories received $(\mathrm{kcal} / \mathrm{kg})$ between the groups. First fecal passage, postoperative albumin infusion, $\Delta$ alb, duration of SIRS and respirator use, and the length of postoperative hospital stay were also comparable between Group D1 and Group D2-3 (Table 3).

\section{Discussion}

In general, esophageal cancer patients are frequently malnourished due to several possible factors including esophageal stenosis, their habits, preoperative systemic chemotherapy, or the systemic effect of their neoplasm [31]. However, preoperative nutritional status was not poor in our study, based on nutritional parameters indicated by BMI, body weight, and serum albumin concentration, showing a goodnutrition preference. In addition, the preoperative nutritional conditions were comparable between groups in this study. Surgical stress in both groups also seemed to be equivalent because there was no significant difference in operative time or blood loss between Group D1 and Group D2-3.

Unlike some previous studies [22, 27], our findings showed no advantage of starting EN within $24 \mathrm{~h}$ after esophagectomy for reducing postoperative morbidity or life-threat- ening complications. However, there was some evidence in the present study of reduced complications with the early EN initiated within $24 \mathrm{~h}$. The discrepancy in morbidity results between past studies and ours might be due to the different nutritional support methods used, in that most of the previous studies compared early EN versus TPN, which might have resulted in the superior results with EN. However, our study compared early EN initiated within 24 h versus EN initiated during $24-72 \mathrm{~h}$, with no apparent differences evident in postoperative morbidity or life-threatening complications between the groups.

In addition, while there was no significant difference in infectious complications among our patient cohort, Group D1 patients showed a significantly higher incidence of pneumonia than those in Group D2-3. EN administered within $24 \mathrm{~h}$ after esophagectomy or pancreatoduodenectomy has resulted in impaired respiratory mechanics indicated by significantly lower vital capacity and forced expiratory volume in one second $\left(\mathrm{FEV}_{1}\right)$ compared with controls [20], and indeed, our study did show a high frequency of recurrent nerve palsy in both groups. However, we believe that the strict endoscopic assessment of recurrent nerve palsy in our department might account for this finding, and that most of the cases were in fact subclinical and transient. Moreover, there was no significant association between recurrent nerve palsy and postoperative pneumonia in our study. Therefore, early EN initiated within $24 \mathrm{~h}$ might also cause impaired respiratory function such as decreased vital capacity and/or FEV $\mathrm{FE}_{1}$, and thus lead to the high frequency of postoperative pneumonia in Group D1 in our study.

Postoperative outcomes indicated by first fecal passage, albumin infusion, difference in serum albumin between pre-operative and day 7 postoperative measurements were comparable between the two groups in our study, as were 
supplied EN calories, the duration of SIRS and respiratory management. Therefore, we found no significant benefits specifically associated with early EN initiated within $24 \mathrm{~h}$ after esopgagectomy compared with that initiated at $24-72 \mathrm{~h}$.

\section{Conclusion}

Administering EN within $24 \mathrm{~h}$ showed no further advantage for the postoperative course of patients undergoing surgery for esophageal cancer compared to EN started within 24 - 72 h. We therefore recommend that early EN initiation should be scheduled according to the condition of the patient in the range of $72 \mathrm{~h}$ after esophagectomy.

\section{Financial Disclosure}

We declare that we have no conflict of interest.

\section{References}

1. Doig GS, Heighes PT, Simpson F, Sweetman EA, Davies AR. Early enteral nutrition, provided within $24 \mathrm{~h}$ of injury or intensive care unit admission, significantly reduces mortality in critically ill patients: a meta-analysis of randomised controlled trials. Intensive Care Med. 2009;35(12):2018-2027.

2. Kompan L, Vidmar G, Spindler-Vesel A, Pecar J. Is early enteral nutrition a risk factor for gastric intolerance and pneumonia? Clin Nutr. 2004;23(4):527-532.

3. Nguyen NQ, Fraser RJ, Bryant LK, Burgstad C, Chapman MJ, Bellon M, Wishart J, et al. The impact of delaying enteral feeding on gastric emptying, plasma cholecystokinin, and peptide YY concentrations in critically ill patients. Crit Care Med. 2008;36(5):1469-1474.

4. Grahm TW, Zadrozny DB, Harrington T. The benefits of early jejunal hyperalimentation in the head-injured patient. Neurosurgery. 1989;25(5):729-735.

5. Taylor SJ, Fettes SB, Jewkes C, Nelson RJ. Prospective, randomized, controlled trial to determine the effect of early enhanced enteral nutrition on clinical outcome in mechanically ventilated patients suffering head injury. Crit Care Med. 1999;27(11):2525-2531.

6. Chiarelli A, Enzi G, Casadei A, Baggio B, Valerio A, Mazzoleni F. Very early nutrition supplementation in burned patients. Am J Clin Nutr. 1990;51(6):1035-1039.

7. Wasiak J, Cleland H, Jeffery R. Early versus delayed enteral nutrition support for burn injuries. Cochrane Database Syst Rev. 2006;(3):CD005489.

8. Chuntrasakul C, Siltharm S, Chinswangwatanakul V, Pongprasobchai T, Chockvivatanavanit S, Bunnak A. Early nutritional support in severe traumatic patients. J Med Assoc Thai. 1996;79(1):21-26.
9. Garrel DR, Davignon I, Lopez D. Length of care in patients with severe burns with or without early enteral nutritional support. A retrospective study. J Burn Care Rehabil. 1991;12(1):85-90.

10. Moore EE, Dunn EL, Jones TN. Immediate jejunostomy feeding. Its use after major abdominal trauma. Arch Surg. 1981;116(5):681-684.

11. Kudsk KA, Croce MA, Fabian TC, Minard G, Tolley EA, Poret HA, Kuhl MR, et al. Enteral versus parenteral feeding. Effects on septic morbidity after blunt and penetrating abdominal trauma. Ann Surg. 1992;215(5):503511; discussion 511-503.

12. Kompan L, Kremzar B, Gadzijev E, Prosek M. Effects of early enteral nutrition on intestinal permeability and the development of multiple organ failure after multiple injury. Intensive Care Med. 1999;25(2):157-161.

13. Moore FA, Feliciano DV, Andrassy RJ, McArdle AH, Booth FV, Morgenstein-Wagner TB, Kellum JM, Jr., et al. Early enteral feeding, compared with parenteral, reduces postoperative septic complications. The results of a meta-analysis. Ann Surg. 1992;216(2):172-183.

14. Heslin MJ, Latkany L, Leung D, Brooks AD, Hochwald SN, Pisters PW, Shike M, et al. A prospective, randomized trial of early enteral feeding after resection of upper gastrointestinal malignancy. Ann Surg. 1997;226(4):567-577; discussion 577-580.

15. Ortiz H, Armendariz P, Yarnoz C. Is early postoperative feeding feasible in elective colon and rectal surgery? Int J Colorectal Dis. 1996;11(3):119-121.

16. Reissman P, Teoh TA, Cohen SM, Weiss EG, Nogueras $\mathrm{JJ}$, Wexner SD. Is early oral feeding safe after elective colorectal surgery? A prospective randomized trial. Ann Surg. 1995;222(1):73-77.

17. Smedley F, Bowling T, James M, Stokes E, Goodger C, O'Connor O, Oldale C, et al. Randomized clinical trial of the effects of preoperative and postoperative oral nutritional supplements on clinical course and cost of care. Br J Surg. 2004;91(8):983-990.

18. Stewart BT, Woods RJ, Collopy BT, Fink RJ, Mackay JR, Keck JO. Early feeding after elective open colorectal resections: a prospective randomized trial. Aust $\mathrm{N} \mathrm{Z} \mathrm{J}$ Surg. 1998;68(2):125-128.

19. Carr CS, Ling KD, Boulos P, Singer M. Randomised trial of safety and efficacy of immediate postoperative enteral feeding in patients undergoing gastrointestinal resection. BMJ. 1996;312(7035):869-871.

20. Watters JM, Kirkpatrick SM, Norris SB, Shamji FM, Wells GA. Immediate postoperative enteral feeding results in impaired respiratory mechanics and decreased mobility. Ann Surg. 1997;226(3):369-377; discussion 377-380.

21. Austrums E, Pupelis G, Snippe K. Postoperative enteral stimulation by gut feeding improves outcomes in severe acute pancreatitis. Nutrition. 2003;19(6):487-491. 
22. Fujita T, Daiko H, Nishimura M. Early enteral nutrition reduces the rate of life-threatening complications after thoracic esophagectomy in patients with esophageal cancer. Eur Surg Res. 2012;48(2):79-84.

23. Moore FA, Moore EE, Jones TN, McCroskey BL, Peterson VM. TEN versus TPN following major abdominal trauma--reduced septic morbidity. J Trauma. 1989;29(7):916-922; discussion 922-913.

24. Jiang K, Cheng L, Wang JJ, Li JS, Nie J. Fast track clinical pathway implications in esophagogastrectomy. World J Gastroenterol. 2009;15(4):496-501.

25. Cao S, Zhao G, Cui J, Dong Q, Qi S, Xin Y, Shen B, et al. Fast-track rehabilitation program and conventional care after esophagectomy: a retrospective controlled cohort study. Support Care Cancer. 2013;21(3):707-714.

26. Weimann A, Braga M, Harsanyi L, Laviano A, Ljungqvist O, Soeters P, Jauch KW, et al. ESPEN Guidelines on Enteral Nutrition: Surgery including organ transplantation. Clin Nutr. 2006;25(2):224-244.

27. Page RD, Oo AY, Russell GN, Pennefather SH. Intrave- nous hydration versus naso-jejunal enteral feeding after esophagectomy: a randomised study. Eur J Cardiothorac Surg. 2002;22(5):666-672.

28. Han-Geurts IJ, Hop WC, Verhoef C, Tran KT, Tilanus HW. Randomized clinical trial comparing feeding jejunostomy with nasoduodenal tube placement in patients undergoing oesophagectomy. Br J Surg. 2007;94(1):3135.

29. Kobayashi K, Koyama Y, Kosugi S, Ishikawa T, Sakamoto K, Ichikawa H, Wakai T. Is early enteral nutrition better for postoperative course in esophageal cancer patients? Nutrients. 2013;5(9):3461-3469.

30. Wheble GA, Benson RA, Khan OA. Is routine postoperative enteral feeding after oesophagectomy worthwhile? Interact Cardiovasc Thorac Surg. 2012;15(4):709-712.

31. Gabor S, Renner H, Matzi V, Ratzenhofer B, Lindenmann J, Sankin O, Pinter H, et al. Early enteral feeding compared with parenteral nutrition after oesophageal or oesophagogastric resection and reconstruction. Br J Nutr. 2005;93(4):509-513. 